\title{
Study of Possible Obstacles Preventing Obtaining a Tokomak Regime in the Egyptian Machine
}

\author{
H. Hegazy, ${ }^{1,2}$ F. Diab, ${ }^{2}$ K. Abd El-Aziz, ${ }^{2}$ Yu. V. Gott, ${ }^{3}$ and M. M. Dremin ${ }^{3}$ \\ ${ }^{1}$ Physics Department, Faculty of Science, Jazan University, Jazan 2097, Saudi Arabia \\ ${ }^{2}$ Plasma Physics Department, NRC, EAEA, Inshas 13759, Egypt \\ ${ }^{3}$ Nuclear Fusion Institute, Russian Research Centre "Kurchatov Institute", Kurchatov Square 1, Moscow 123182, Russia
}

Correspondence should be addressed to H. Hegazy, hos_heg@yahoo.com

Received 25 October 2009; Revised 29 April 2010; Accepted 18 June 2010

Academic Editor: Atsushi Mase

Copyright (c) $2010 \mathrm{H}$. Hegazy et al. This is an open access article distributed under the Creative Commons Attribution License, which permits unrestricted use, distribution, and reproduction in any medium, provided the original work is properly cited.

\begin{abstract}
The main goal of this study is to clarify the possibility to obtain the plasma discharge and to prolong its duration as much as possible, and to analyze what are the preventing reasons of obtaining this goal. Possible reasons can be improper operation of the power supply system, a high level of stray magnetic fields, lack of equilibrium, influence of MHD instabilities, and a high level of impurities. We analyze experimentally these possibilities and discuss them in more details.
\end{abstract}

\section{Introduction and Experimental Facility}

The Egyptian facility is a small device of rectangular cross section $25 \times 20 \mathrm{~cm}$. Its major $(R)$ and minor $(A)$ radius are 30 and $10 \mathrm{~cm}$, respectively. The aspect ratio is 3 . All the necessary electrical circuitry descriptions and its main parameters are given in [1].

The duration of the toroidal field pulse is long enough ( $\sim 30 \mathrm{~ms}$ with charging half of its capacitor bank), so the time interval with relatively small $(\sim 20 \%)$ variation of the toroidal field is about $10 \mathrm{~ms}$. That is why first of all we checked the operation of the ohmic heating power supply system. With the existing circuitry, it critically depends on the normal operation of a Vacuum Interrupter (VI) in the circuit of the so-called OH "slow" battery [1]. With an old VI there was no real interruption of the current because of arcing induced by poor vacuum internally. The installation of a new interrupter would take a lot of time (according to references available in EGYPTOR Tokamak Project Laboratory, a similar system on the COMPASS tokamak was operating correctly after six months of adjustment using special equipment not available in this laboratory) [2].

Because without the VI the OH "slow" battery could not give the loop voltage necessary for breakdown the discharge, we were forced to obtain the discharges with the help of only the $\mathrm{OH}$ "fast" battery which could provide a discharge duration of only $\sim 1 \mathrm{~ms}$. For simultaneous operation of both batteries the tokamak power supply system must be modified. For instance, the system described in [3] can be used.

\section{Results and Discussions}

2.1. Compensation of Stray Magnetic Fields. As the first step, the level of compensation of stray magnetic field with the help of permanent coils should be tested. The main sources of this field could be the toroidal field coils (due to their small misalignment) and the ohmic heating winding (because there is no iron core).

For measurements of the vertical magnetic field from the $\mathrm{OH}$ coil, a pick-up coil was used [1]. This coil was placed at the center of the plasma chamber. In Figure 1, one can see the wave-form of the stray magnetic field from the $\mathrm{OH}$ coil without compensation. The wave-form of this magnetic field with compensation is presented in Figure 2.

So one can see that the compensation reduces the stray magnetic field about 4.6 times. Taking into account that the sensitivity of the pick-up coil is $B[T]=0.017 U[V]$ (where $B$ is the magnetic field value and $U$ is the signal value), and that in these experiments the voltage on the $\mathrm{OH}$ battery was $1 \mathrm{kV}$, one can find that the value of the stray magnetic field 


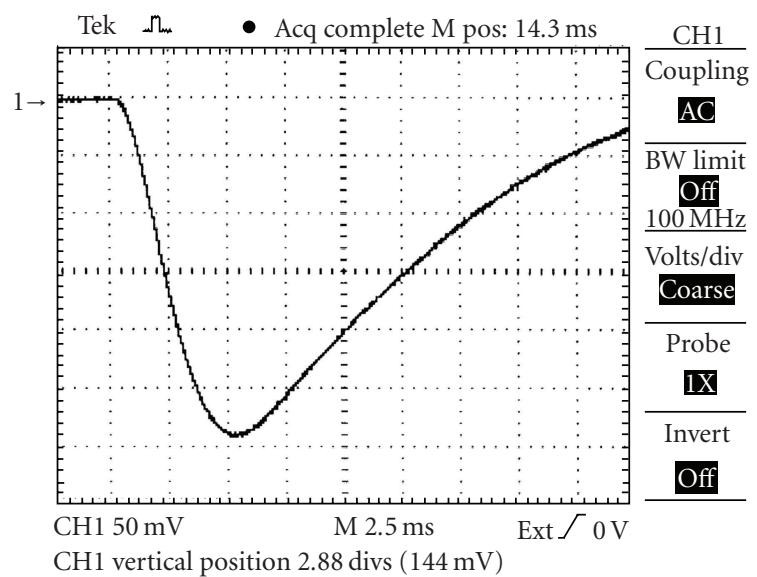

FIGURE 1: Wave-form of vertical field from $\mathrm{OH}$ coil without compensation.

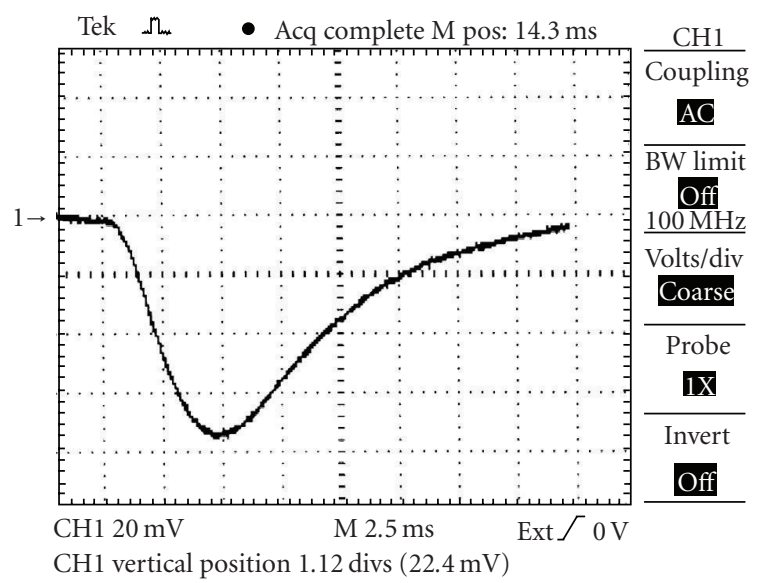

FIGURE 2: Wave-form of vertical field from $\mathrm{OH}$ coil with compensation.

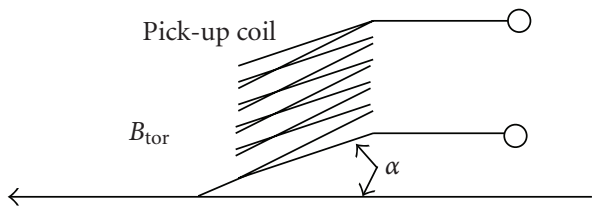

Figure 3: Position of the pick-up coil for stray magnetic field measurement.

after compensation is about $1.2 \times 10^{-3} U_{\mathrm{OH}}[k V]$, where $U_{\mathrm{OH}}$ is the $\mathrm{OH}$ battery voltage in $\mathrm{kV}$.

The measurement of the vertical component of the toroidal magnetic field with the help of the same pick-up coil is practically impossible because it is very difficult to place this coil properly (see Figure 3 ).

The plane of the pick-up coils must be parallel to the toroidal magnetic field $B_{\text {tor }}$. If this condition is not met, a signal will be generated in the pick-up coil, which is

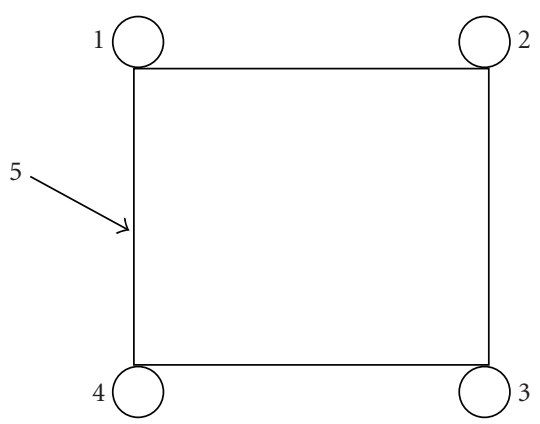

Figure 4: The coils for the measurements of toroidal magnetic field vertical component. 1-4: additional coils, 5: vacuum chamber.

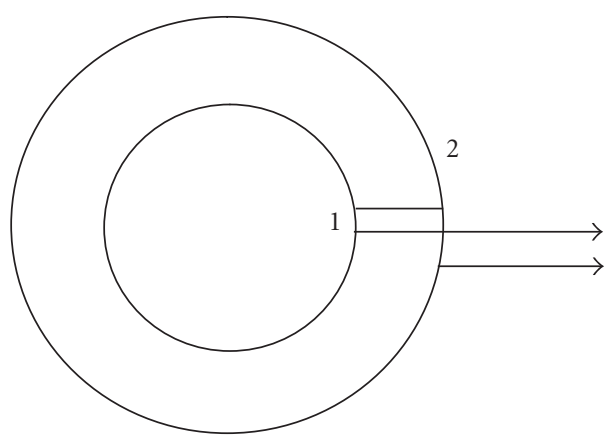

FIGURE 5: The 1-2 coils connections.

proportional to $\sin \alpha$. In practice the value of this signal is much greater than the signal from the stray magnetic field.

For measurements of the stray vertical field of the toroidal field coils, four (1-4) additional coils were used. These coils were placed on the bottom and top sides of the vacuum chamber as is shown in Figure 4.

Coil 1 was connected with coil 2 in series as it can be seen in Figure 5.

Coil 3 was connected with coil 4 in series, too. The difference between signals from loops 1 and 2 (or 3 and 4), which can be obtained after subtraction (or summation with opposite polarity) on a resistive divider, gives after integration the value of the vertical magnetic flux $\Psi_{1-2}$ or $\Psi_{3-4}$ between these two loops. Knowing their dimensions (radii) and, accordingly, the surface between them, $S_{1-2}=$ $\pi\left(R_{1}^{2}-R_{2}^{2}\right)$, one can find the average vertical magnetic field $B_{\perp}=\Psi_{1-2} / \pi\left(R_{1}{ }^{2}-R_{2}{ }^{2}\right)$. Absolute values of $B_{\perp}$ field can be determined by comparison with measurements by a calibrated pick-up coil [3]. Since the effective surface of this coil equals to $\pi D^{2} N(D=1.5 \mathrm{~cm}$ is its diameter, $N=$ 120: number of turns), that is, $842 \mathrm{~cm}^{2}$, and surface $S_{1-2}$ equals to $\pi\left(45^{2}-15^{2}\right)=5655 \mathrm{~cm}^{2}$, so with using the same integrator their sensitivities will differ by a factor 6.7 (loops are more sensitive). So the sensitivity of loops $1-2$ is equal to $0.114 \mathrm{~T} / \mathrm{V}$. The waveform of the vertical component of the toroidal magnetic field is given in Figure 6.

In a similar manner, one can obtain estimations of the average horizontal magnetic field which determines the equilibrium in vertical direction. For this purpose one should 


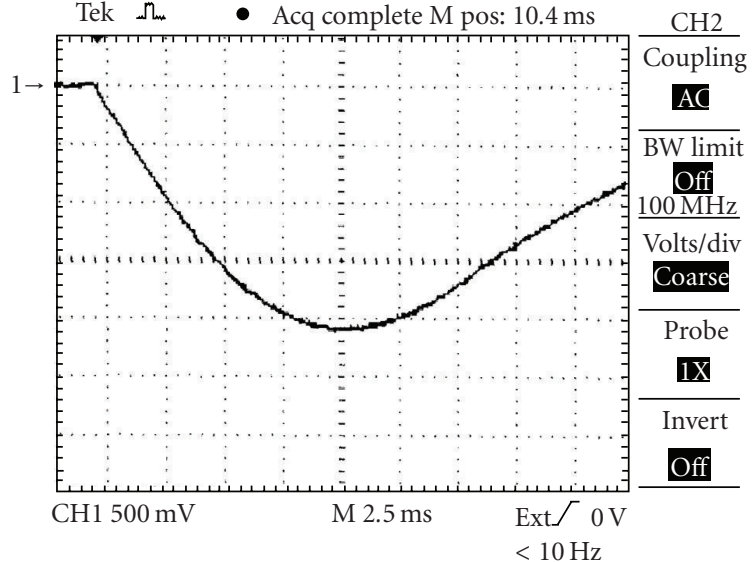

Figure 6: The vertical component of the toroidal magnetic field measured with the help of coils $1+2$.

combine the signals of loops 1 and 3 (or 2 and 4), but their effective surface in this case will be $S_{1-3}=2 \pi R_{1,3} \Delta z$, where $\Delta z$ is vertical distance between loops 1 and $3(38 \mathrm{~cm})$, that is, $S_{1-3}=10744 \mathrm{~cm}^{2}$.

The signal from $1+2$ coils is given in Figure 6. Taking into account the radii of the inner and outer coils (15 and $45 \mathrm{~cm}$ ), we can estimate the value of the stray vertical fields from toroidal coil and ohmic heating coil as being not higher than $5 \mathrm{Gs}$. So we can conclude that the measured values of stray magnetic fields cannot prevent the discharge breakdown and limit its duration.

2.2. Plasma Equilibrium. Next we studied the plasma column equilibrium. For these we used the pair of Mirnov probes (outer and inner) installed in the vacuum chamber. The precise evaluation of the plasma position in the chamber envisage the calibrated measurements of poloidal magnetic field and average vertical magnetic field in accordance with the formulae

$$
\begin{aligned}
\frac{\Delta}{b}=\frac{b}{2 R}\left[\frac{a^{2}}{b^{2}} \ln \frac{b}{a}-\frac{1}{2}\left(1-\frac{a^{2}}{b^{2}}\right)\right] & \\
+\frac{c b}{4 J} & {\left[\frac{B_{\omega+(1+b / R)}-B_{\omega-(1-b / R)}}{2}\left(1-\frac{a^{2}}{b^{2}}\right)\right.} \\
& \left.-B_{\perp}\left(1+\frac{a^{2}}{b^{2}}\right)\right],
\end{aligned}
$$

where $\Delta$ is horizontal displacement of the plasma column, $a$ is minor plasma radius, $R$ is major plasma radius, $b$ is minor radius on which the Mirnov probes are placed, $J$ is plasma current, $B_{\omega+}$ and $B_{\omega-}$ is azumuthal magnetic field measured by outer and inner Mirnov probes accordingly, $B_{\perp}$ is averaged transverse magnetic field measured by loops $1-2$ or 3-4 (see Figure 7).

Because Mirnov probes and these loops were not calibrated, we could make only some estimations of the plasma equilibrium. If the center of the plasma current coincides with the center of the chamber (i.e., at equal distances from
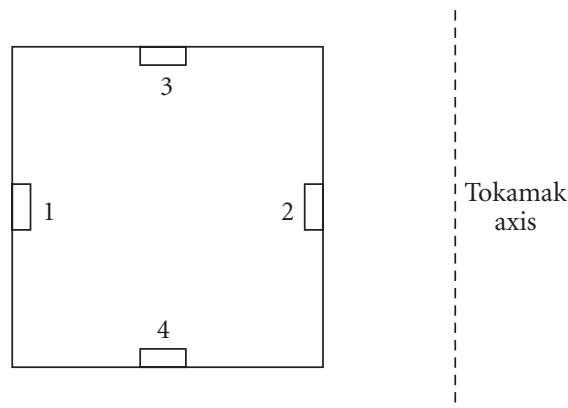

FIGURE 7: Mirnov's probes locations.

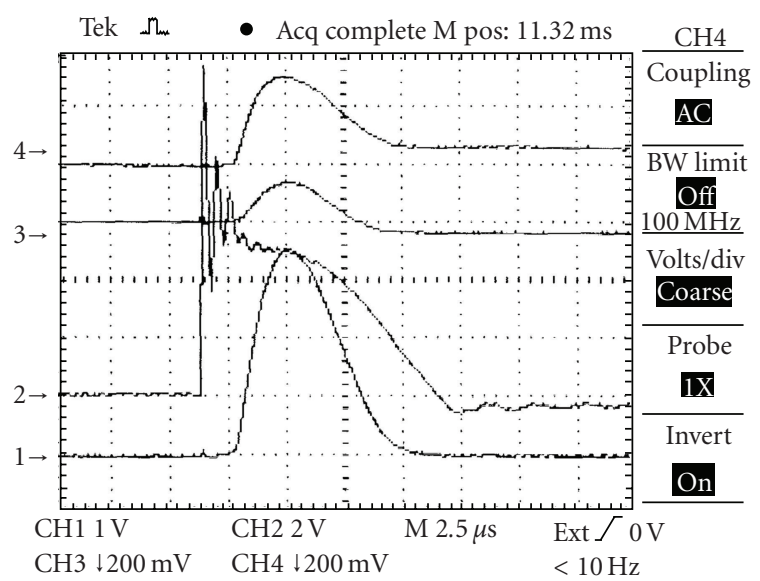

Figure 8: The signals from Mirnov's coils: coil 1 Ch 3 and coil 2 Ch 4, plasma current Ch 1, and loop voltage Ch 2.

both probes) these signals must be equal (in cylindrical approximation). In a torus these signals will differ due the toroidicity by the ratio $(R+b) /(R-b)$. Due to the lack of equilibrium, that is, displacement $\Delta$ of plasma current relative to chamber axis, the signal in one of Mirnov probes (outer) will increase $\sim J_{p} /(b-\Delta)\left(\left(J_{p} / b\right)(1+\Delta / b)\right.$ for small displacements). Analogously, the signal from inner probe will decrease as $\left(J_{p} / b\right)(1-\Delta / b)$ and their difference will be $\sim 2 J_{p} \Delta / b^{2}$. So if we adjust the signals from these two Mirnov probes in accordance with their toroidicity and then subtract these signals, we will obtain the signal proportional to displacement of the plasma current. But as one can see from these signals (Figure 8) their shapes are similar and repeat practically the shape of plasma current signal.

The relative difference between these signals (or signal after their normalization and subtraction-Figure 9) is not more than 0.125 which corresponds to displacement of not more than $0.06 \mathrm{~b}$, that is, $\sim 0.5 \mathrm{~cm}$.

So we can conclude that the plasma equilibrium in these discharges is good enough and in any case could not be the reason for their short duration or small plasma current value.

2.3. MHD Instabilities. The value and duration of the plasma current can be, in principle, limited due to the development of MHD instabilities. Conditions for this development are characterized by the $q$ parameter which is determined 


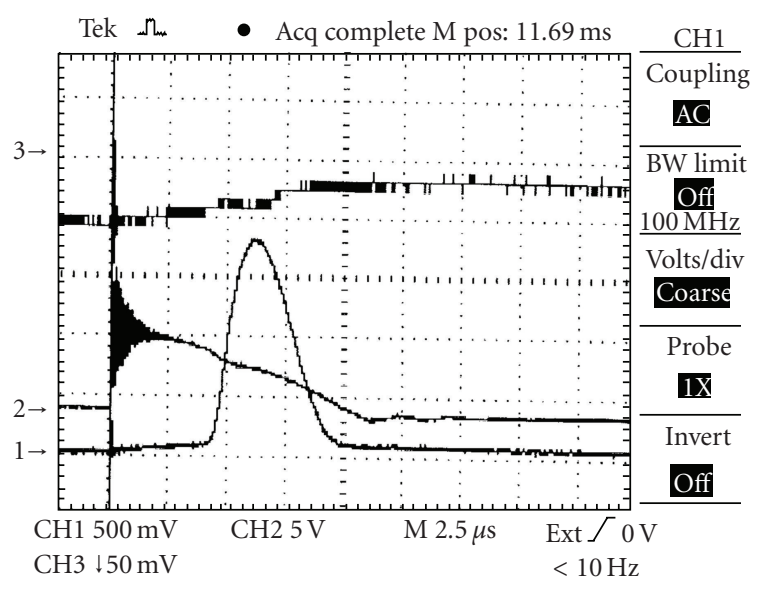

Figure 9: Relative difference between signals from two Mirnov's coils Ch.(3), plasma current Ch (1), and loop voltage Ch.(2) 1Plasma current, 2-loop voltage.

as $\left(B_{t} / B_{\omega}\right)(a / R)$, where $B_{t}$ is toroidal magnetic field and $B_{\omega}[\mathrm{Gs}]=2 \cdot 10^{-5} J_{p}[\mathrm{~A}] / a[\mathrm{~cm}]$ —azimuthal field of plasma current. When it equals some small integral values the rational surfaces exist in the plasma, magnetic islands can develop and plasma confinement can deteriorate. Keeping in mind that $R=30 \mathrm{~cm}$, we obtain, for $q$,

$$
q=\frac{1.7 \cdot 10^{3} B_{t}[\mathrm{~T}] a^{2}[\mathrm{~cm}]}{I_{p}[\mathrm{~A}]}
$$

For $B_{t}=0.4 \mathrm{~T}$ (corresponding to $U_{\text {tor }}=1 \mathrm{kV}$ ), plasma current $J_{p}=5 \mathrm{kA}$, and $a=7 \mathrm{~cm}$, the $q$ value equals to $1.7 \cdot 10^{3}$. $0.4 \cdot 49 / 5 \cdot 10^{3}=6.7$. This value is large enough because most dangerous MHD modes have $q$ values of 2 and 3. So MHD instabilities most likely cannot be responsible for the short duration and the small amplitude of the plasma current.

2.4. Influence of Impurities. As the next step, we tried to analyze the influence of impurities. Due to lack of measurements of radiated power and impurities spectral line intensities, we made estimations of plasma electron temperature $T_{e}$. They were based on the dependence of plasma resistivity $\eta$ on $T_{e}$ expressed by formulas [4]

$$
\begin{gathered}
\eta_{H}=1.65 \cdot 10^{-9} \frac{\ln \Lambda}{T_{e}^{3 / 2}} \mathrm{Ohm} \cdot \mathrm{m}, \quad T_{e} \text { in } \mathrm{keV}, \\
\eta_{Z}=N(Z) \cdot Z \cdot \eta_{H} .
\end{gathered}
$$

Knowing the plasma resistance from the plasma current $J_{p}$ and the loop voltage $U$ (taken in the moment of maximum plasma current when the inductive component of loop voltage $U_{L}=L_{p} \partial J_{p} / \partial t, L_{p}$ is the inductance of plasma column) equals zero due to $\left(\partial I_{p} / \partial e=0\right) ; R=U / J_{p}$ one can estimate the plasma resistivity $\eta=R \cdot S / l$, where $S$ is plasma-cross section $\pi a^{2}$ ( $a$ is plasma minor radius which usually can be taken as limiter radius), $l=2 \pi R$ is length of plasma axis (here $R=0.3 \mathrm{~m}$ is the plasma major radius). The value of Coulomb logarithm $\ln \Lambda$ is weakly dependent on plasma density and can be taken as 17 . Parameter $N$ is weakly dependent on the effective charge of plasma ions $Z$ and with the assumption that the main impurity is carbon $(Z \approx 5)$ can be taken as 0.72 . With these parameters we obtain

$$
\begin{aligned}
& \eta=\frac{U[\mathrm{~V}] a^{2}[\mathrm{~m}]}{0.6 J_{p}[\mathrm{~A}]}=\frac{1.01 \cdot 10^{-7}}{T_{e}^{3 / 2}[\mathrm{keV}]}, \\
& T_{e}[\mathrm{keV}]=1.54 \cdot 10^{-5}\left(\frac{J_{p}}{U a^{2}}\right)^{2 / 3} .
\end{aligned}
$$

For a plasma current $J_{p}=5 \mathrm{kA}$ and a loop voltage $25 \mathrm{~V}$ and assuming $a=7 \mathrm{~cm}$, we obtain $T_{e}=1.54$. $10^{-5}\left(5 \cdot 10^{3} / 25 \cdot 49 \cdot 10^{-4}\right)^{2 / 3} \approx 20 \mathrm{eV}$. This value is very close to the so-called "radiation limit" which was observed in first tokamaks and is associated with high level of impurities.

\section{Counclusion}

As an obvious way to improve the plasma performance in the EGYPTOR tokamak, we propose decreasing the level of plasma impurities and conditioning the chamber walls by discharge cleaning ( $50 \mathrm{~Hz}$ Taylor type and inductive) under the corresponding control of impurities content with the help of measurements of radiation power and/or spectroscopic impurity (carbon and oxygen first of all) behavior.

Moreover, for simultaneous operation of both $\mathrm{OH}$ "fast and slow" batteries, the tokamak power supply system must be modified. For instance, the system described in [3] can be used as it was seen to be valuable in other small Tokamaks.

\section{References}

[1] H. Hegazy and F. Zacek, "Calibration of power systems and measurements of discharge currents generated for different coils in the EGYPTOR tokamak," Journal of Fusion Energy, vol. 25, no. 1-2, pp. 73-86, 2006.

[2] D. C. Edvards, P. M. Barnes, J. H. Ray, et al., Compass Electrical Systems Development and Commissioning, UKAEA Culham Laboratory, Oxon, UK.

[3] M. Valovič, "An ohmic heating circuit for the CASTOR tokamak," Czechoslovak Journal of Physics, vol. 40, no. 6, pp. 673-677, 1990.

[4] J. Wesson, Tokamaks, Clarendon Press, Oxford, UK, 2004. 